\title{
Prevalência e fatores associados a níveis insuficientes de atividade física em jovens estudantes de duas cidades brasileiras: últimos sete dias e semana típica ou normal
}

CDD. 20.ed. 613.7

\author{
J osé Cazuza de FARIAS J ÚNIOR* \\ Fernando Vinholes SIQUEIRA* \\ Markus Vinicius NAHAS ${ }^{* * *}$ \\ Mauro Virgúlio Gomes de BARROS ${ }^{* * * *}$
}

\section{Resumo}

Este estudo comparou as prevalências de níveis insuficientes de atividade física e fatores associados determinados a partir da medida das atividades físicas praticadas nos últimos sete dias e durante uma semana típica ou normal. Trata-se de uma análise transversal dos dados de base ("baseline data") de uma intervenção escolar randomizada (Projeto Saúde na Boa), que incluiu 2.096 jovens (15-24 anos; 55,6\% do sexo feminino), de 20 escolas públicas (10 em Florianópolis e 10 em Recife), pareadas por tamanho e localização geográfica. Os dados foram coletados por meio de questionário previamente validado. Foram classificados como "insuficientemente ativos" os sujeitos que não atingiram dose recomendada de prática de atividades físicas ( $\geq 60$ minutos diários de atividades físicas de intensidade moderada a vigorosa, $\geq$ 5dias/semana). A medida de atividade física foi efetuada considerando dois períodos de referência: os últimos sete dias e uma semana típica ou normal. A medida das atividades físicas praticadas considerando a referência dos últimos sete dias resultou em prevalência mais elevada de níveis insuficientes de atividade física em comparação à medida considerando a referência da semana típica ou normal $(60,8 \%$ vs. $54,6 \% ; p<0,001)$. Independentemente do período de referência da medida de atividade física, foram identificados os mesmos fatores associados a níveis insuficientes de atividade fisica, com uma discreta variação na magnitude das medidas de associação desse desfecho com os fatores analisados. Utilizar os últimos sete dias ou uma semana típica como referência para mensurar as atividades físicas parece produzir prevalências de níveis insuficientes de atividade física diferentes, mas fatores associados semelhantes.

UnITERMos: Jovens; Medida da atividade física; Prevalência; Fatores associados.

\section{Introdução}

Em estudos epidemiológicos, independentemente do grupo populacional, a medida do nível de atividade física ainda continua sendo efetuada, essencialmente, por meio de questionário (HALLAL, Dumith, Bastos, Reichert, Siqueira \& Azevedo, 2007; Varo, Martinez-González, Irala-Estévez, Kearney, Gibney \& Martinez, 2003). Isso se deve ao baixo custo, a praticidade e a possibilidade de mensurar, de forma simultânea, diferentes parâmetros da atividade física (tipo, frequência, duração e intensidade das atividades físicas praticadas em um ou mais contextos: lazer, deslocamento, lar, trabalho, escola) (Kohl, Fulton \& Caspersen, 2000; Sirard \& PATE, 2001).

Há uma grande diversidade de questionários para mensurar os níveis de atividade física (ARMSTRONG \& Welsman, 2006; Sallis \& Saelens, 2000). Dentre outros aspectos, estes instrumentos diferem em relação ao contexto da medida (lazer, deslocamento, lar, trabalho), tamanho (número de questôes) e ao período de recordação das atividades praticadas (um dia, uma semana, mês, ano) (CHINAPAW, MOKKINK, Van Poppel, Van Mechelen \& Terwee, 2010; Kohl, Fulton \& Caspersen, 2000; Sallis \& Saelens, 2000). 
Nos últimos anos, tem crescido a utilização de questionários "simplificados" em estudos epidemiológicos para descrever a prevalência de níveis insuficientes de atividade física (CDC, 2008; WHO, 2008), identificar fatores associados à atividade física (Butcher, Sallis, Mayer \& Woodruff, 2008) e avaliar o impacto de programas de intervenção sobre atividade física (Barros, Nahas, Hallal, Farias Júnior, Florindo \& Honda, 2009; Prochaska, Zabinski, Calfas, Sallis \& Patrick, 2000). Estes instrumentos, são compostos por uma ou duas questóes, mensuram a frequência de prática de atividade física moderadas a vigorosa, num determinado período de tempo (por exemplo, $\geq 60 \mathrm{minutos} / \mathrm{dia}$ ) e têm demonstrado níveis satisfatórios de reprodutibilidade (coeficiente de correlação intraclasse: 0,88; kappa $=61 \%)$ e validade ( $(\mathrm{ho}=0,40$; sensibilidade $=71 \%$, comparado a medida de acelerômetros) (Prochaska, Sallis \& Long, 2001).

Não há consenso em relação ao período de referência que deve ser adotado quando se mede o nível

\section{Métodos}

Trata-se da análise transversal dos dados de base ("baseline data") de um estudo de intervenção denominado "Projeto Saúde na Boa"(NAHAS, Barros, Assis, Hallal, Florindo \& Konrad, 2009), que incluiu estudantes do ensino médio noturno, de escolas públicas estaduais, de Florianópolis, SC e Recife, PE.

Foram analisados dados de 2.096 jovens, de 15 a 24 anos de idade, de 20 escolas (10 em cada município), selecionadas aleatoriamente, após pareamento por tamanho (pequena até 200 alunos; média de 200 a 499 alunos; e grande de 500 ou mais alunos) e localização geográfica. Informações detalhadas sobre a população alvo e o procedimento de seleção da amostra estão descritas em publicações prévias (Barros et al., 2009; Nahas, Barros, Goldfine, Lopes, Hallal, Farias Júnior \& Oliveira, 2009).

Para fins deste estudo, o cálculo do poder estatístico da amostra foi realizado "a posteriori". Com um $\mathrm{n}=2.096$ é possível estimar prevalências iguais ou superiores a $20 \%$ com erro de três pontos percentuais e nível de confiança de $95 \%$, e detectar uma razão de prevalência igual ou superior a 1,15 como estatisticamente significativa, $\operatorname{com} \alpha=5 \%$ e $1-\beta=$ $80 \%$, com prevalência do desfecho nos expostos igual ou superior a $40 \%$. de atividade física por questionário, sobretudo os simplificados. Enquanto alguns estudos utilizam os últimos sete dias ou a última semana, outros recorrem a uma semana típica ou normal (ARMSTRONG $\&$ WelsMan, 2006; SALLIS \& SAELENS, 2000; Sirard $\&$ PATE, 2001). Em tese, esse dois períodos de recordação refletem aspectos distintos da prática de atividade física. O primeiro representa uma medida das atividades físicas atuais ou recentes e o segundo as atividades habitualmente praticadas.

Pouco se sabe sobre a possibilidade de ocorrer diferenças nas prevalências de níveis insuficientes de atividade física e nos fatores associados ao se recorrer aos últimos sete dias ou uma semana típica como período de referência para recordar as atividades praticadas. Sendo assim, o presente estudo foi desenvolvido com intuito de comparar as prevalências de níveis insuficientes de atividade física e alguns fatores associados determinados a partir da medida das atividades físicas praticadas durante os últimos sete dias e em uma semana típica ou normal.

Em março de 2007, os estudantes responderam o questionário "Saúde na Boa", previamente validado (Nahas, Barros, Florindo, Farias Junior, Hallal, Konrad, Barros \& Assis, 2007). O instrumento foi aplicado em sala de aula, seguindo instruções prévias, fornecidas por equipe previamente treinada sob o mesmo protocolo, em ambos os municípios. Este questionário incluía itens sobre atividade física e hábitos alimentares, baseados no projeto PACE+ (Patient-Centered Assessment and Counseling for Exercise Plus Nutrition) (NAHAS et al., 2007; ProCHASKA et al., 2000), comportamentos sedentários, fumo, atitude em relação à prática de atividade física, estágio de mudança de comportamento, percepção de saúde, orientação para prática de atividade física e questões sociodemográficas. Em subamostra com características similares as do presente estudo essas variáveis apresentaram índice concordância kappa entre 0,36 e 0,96.

No presente estudo, foram analisados os seguintes desfechos: a) atividade física nos últimos sete dias que antecederam a coleta de dados ("Nos últimos sete dias, em quantos dias você fez atividades físicas que, somadas, totalizam ao menos 60 minutos por dia?”); b) atividade física em uma semana típica ou normal ("Durante uma semana típica ou normal, 
em quantos dias você faz atividades físicas que, somadas, totalizam ao menos 60 minutos por dia?"). Nas duas questões foram utilizadas um escala de medida que variava de nenhum dia/semana a sete dias/semana. Estas duas questões demonstraram níveis elevados de reprodutibilidade $(0,87$ a 0,93$)$ e validade concorrente moderada $(0,37$ a 0,41$)$ (NAHAs et al., 2007; Prochaska et al., 2000).

Os sujeitos que relataram participar de pelo menos 60 minutos de atividades físicas moderadas a vigorosas, durante cinco ou mais dias por semana foram classificados como "fisicamente ativos", e os demais foram denominados "insuficientemente ativos" (Biddle, Sallis \& Cavill, 1998; Strong, Malina, Blimkie, Daniels, Dishman, Gutin, Hergenroeder, Must, Nixon, Pivarnik, Rowland, Trost \& Trudeau, 2005).

As variáveis independentes foram: a) sexo (masculino e feminino); b) idade (15-19 anos e 20-24 anos); c) cor da pele (branca e não-branca); d) tabagismo (sim ou não); e) situação conjugal (solteiro ou outros); f) trabalhador (sim ou não); g) reside com a família (sim ou não); h) tipo de moradia (casa, apartamento/outro); i) recebeu aconselhamento, no último ano escolar, para praticar atividade física (sim ou não); e j) percepção de saúde (boa/excelente e regular/ruim).

As análises descritivas incluíram cálculos de proporçóes e respectivos intervalos de confiança (IC95\%). O teste de McNemar foi utilizado para comparar as prevalências de níveis insuficientes de atividade física entre a medida de atividade física nos últimos sete dias e durante uma semana típica ou normal.

\section{Resultados}

Dos 2.096 jovens (média de idade $=18,4 ; \mathrm{DP}$ $=2,3)$ que participaram do estudo $(53,7 \%$ de Florianópolis, SC), a maioria era do sexo feminino (55,6\%), tinha $15-19$ anos de idade $(72,9 \%)$, referiu ter a cor da pele não-branca $(57,4 \%)$, era solteiro
$\mathrm{Na}$ análise bruta, foi verificada a prevalência de níveis insuficientes de atividade física para cada uma das variáveis independentes (letras de a-j). $\mathrm{Na}$ análise ajustada, foi utilizada a regressão de Poisson, com variância robusta (BARROS \& HirAKATA, 2003). Para a determinação dos níveis de significância foi utilizado o teste de Wald.

A análise ajustada seguiu um modelo hierárquico de determinação do desfecho, considerando cinco níveis de entrada das variáveis no modelo, conforme recomendações da literatura (Victora, HutTly, Fuches \& Olinto, 1997). No primeiro nível foram utilizadas as variáveis de "a-c". No segundo nível a variável " $d$ ". No terceiro nível as variáveis "e-g", enquanto o quarto nível foi composto pela variável " $h$ ". O quinto e sexto níveis foram formados pelas variáveis “i” e “j”, respectivamente. Variáveis com valor de $\mathrm{p}<0,20$ foram mantidas no modelo de análise para controle de possíveis fatores de confusão (Maldona \& GreEland, 1993). Não foram observadas interaçóes significativas entre sexo e as demais variáveis independentes. Sendo assim, a análise não foi estratificada por sexo. Todas as análises estatísticas foram realizadas no Stata 11 e levaram em consideração a estratégia de seleção da amostra por conglomerados (opção svy do Stata). O nível de significância adotado foi 5\% para testes bicaudais.

Os Comitês de Ética da Universidade Federal de Santa Catarina (031/2005) e do Instituto Materno Infantil de Pernambuco (587/2005) aprovaram o protocolo deste estudo. Foi obtido o termo de consentimento negativo ("parental passive consent form") dos pais ou responsáveis pelos estudantes menores de 18 anos e diretamente no caso dos estudantes com 18 anos ou mais.

$(87,5 \%)$, informou que não recebeu, no último ano escolar, aconselhamento para praticar atividade física $(56,6 \%)$ e percebeu a sua condição de saúde como boa/excelente $(65,7 \%)$. Outras características da amostra estão descritas na TABELA 1. 
TABELA 1 - Descrição da amostra para o estudo das medidas de atividade física em jovens do ensino médio de Florianópolis, SC e Recife, PE, 2007.

\begin{tabular}{|c|c|c|}
\hline Variáveis & Amostra (\%) & $\mathbf{n}$ \\
\hline Sexo & & 2.088 \\
\hline Masculino & 44,4 & \\
\hline Feminino & 55,6 & \\
\hline Idade & & 2.096 \\
\hline 15 a 19 anos & 72,9 & \\
\hline 20 a 24 anos & 27,1 & \\
\hline Cor da pele & & 2.087 \\
\hline Branca & 42,6 & \\
\hline Não-branca & 57,4 & \\
\hline Tabagismo & & 2.068 \\
\hline Não & 82,0 & \\
\hline Sim & 18,0 & \\
\hline Situação conjugal & & 2.087 \\
\hline Solteiro (a) & 87,5 & \\
\hline Outros & 12,5 & \\
\hline Trabalhador & & 2.084 \\
\hline Não & 53,8 & \\
\hline Sim & 47,2 & \\
\hline Reside com a família & & 2.089 \\
\hline Não & 11,1 & \\
\hline Sim & 88,9 & \\
\hline Tipo de moradia & & 2.088 \\
\hline Casa & 89,3 & \\
\hline Apartamento/Outros & 10,7 & \\
\hline Aconselhamento para atividade física & & 2.071 \\
\hline Não & 56,6 & \\
\hline Sim & 44,4 & \\
\hline Percepção da saúde & & 2.076 \\
\hline Boa/Excelente & 65,7 & \\
\hline Regular/Ruim & 34,3 & \\
\hline
\end{tabular}

A prevalência de níveis insuficientes de atividade física foi mais elevada quando a medida de atividade física levou em consideração as atividades praticadas nos últimos sete dias em comparação à medida considerando uma semana típica ou normal (FIGURA 1), na amostral total (60,8\%; IC95\%:
58,7-62,9 vs. 54,6\%; IC95\%: 52,5-56,8; p < $0,001)$ e em ambos os municípios (Florianópolis, SC: 62,8\%; IC95\%: 59, 9-65,7 vs. 56,9\%; IC95\%: 53,9-59,8; p < 0,001 e Recife, PE: 58,5\%; IC95\%: 55,4-61,7 vs. 52,0\%; IC95\%: 48,9-55,2; p < $0,001)$. 


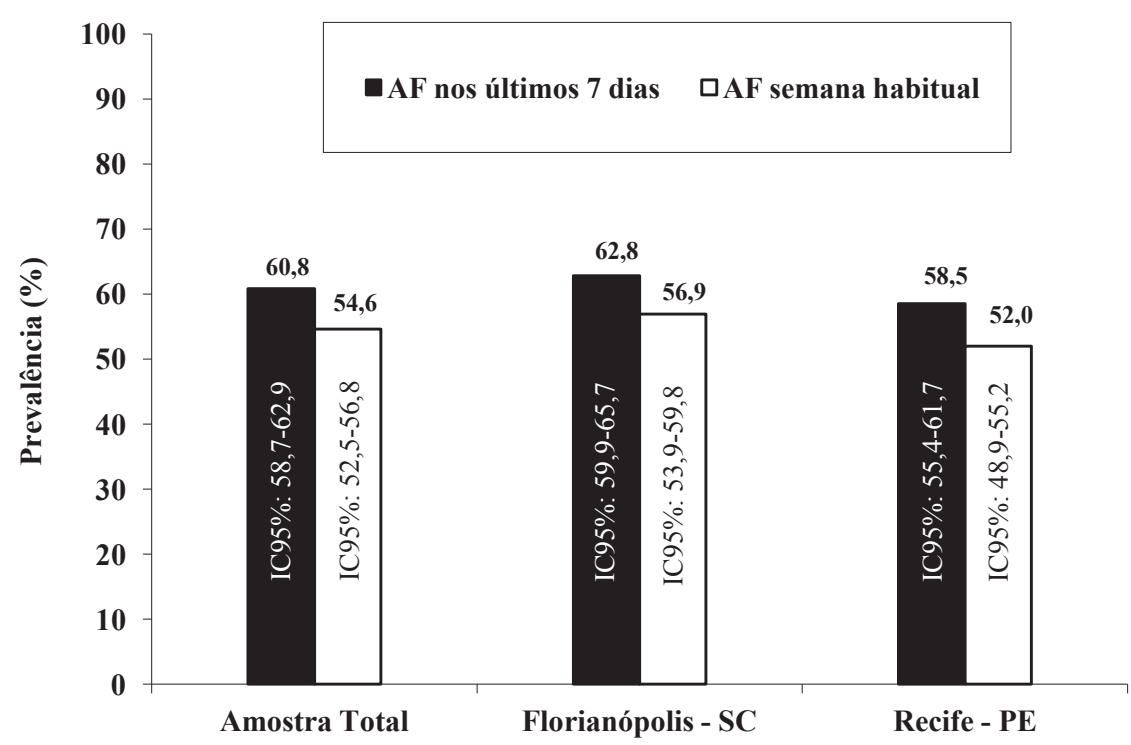

FIGURA 1 - Prevalência deníveis insuficientes deatividadefísica em jovens do ensino médio noturno considerando medida das atividades físicas praticadas nos últimos sete dias e durante uma semana típica ou normal, na amostra total e por município (Florianópolis, SC e Recife, PE), 2007.

Na TABELA 2 estão descritos os resultados da análise bruta da associação entre níveis insuficientes de atividade física e as variáveis independentes. Verificou-se que os fatores associados a níveis insuficientes de atividade física foram similares para as duas medidas: sexo, cor da pele, trabalho e percepção de saúde.

$\mathrm{Na}$ análise ajustada (TABELA 3), mantiveram-se associadas a níveis insuficientes de atividade física as mesmas variáveis identificadas na análise bruta, com ligeiras reduções nas magnitudes das medidas de associação nas variáveis sexo e percepção de saúde, sendo o comportamento semelhante em ambas as medidas.

Não foram encontradas diferenças significativas (interseção dos IC95\% das razões de prevalência) nas magnitudes das medidas de associação entre níveis insuficientes de atividade física e os fatores associados para as medidas de atividade física nos últimos sete dias e durante uma semana típica normal (TABELA 3). Resultados semelhantes também foram encontrados mesmo após estratificar a análise por município (dados não publicados). 
TABELA 2 - Prevalência e análise bruta para as medidas de níveis insuficientes de atividade física em jovens do ensino médio noturno de Florianópolis, SC e Recife, PE, 2007.

*Teste de Wald para heterogeneidade; $\mathrm{RP}=$ razão de prevalência.

\begin{tabular}{|c|c|c|c|c|c|c|}
\hline \multirow{2}{*}{ Variáveis } & \multicolumn{3}{|c|}{ AF nos últimos sete dias } & \multicolumn{3}{|c|}{ AF na semana típica ou normal } \\
\hline & $\%$ & RP (IC95\%) & $\mathbf{p}^{*}$ & $\%$ & RP (IC95\%) & $\mathbf{p}^{*}$ \\
\hline Sexo & & & $<0,001$ & & & 0,002 \\
\hline Masculino & 55,7 & 1 & & 50,7 & 1 & \\
\hline Feminino & 64,7 & $1,16(1,08-1,25)$ & & 57,5 & $1,13(1,05-1,23)$ & \\
\hline Idade & & & 0,319 & & & 0,122 \\
\hline 15 a 19 anos & 61,5 & 1 & & 55,7 & 1 & \\
\hline 20 a 24 anos & 59,0 & $0,96(0,89-1,04)$ & & 51,8 & $0,93(0,85-1,02)$ & \\
\hline Cor da pele & & & 0,002 & & & 0,015 \\
\hline Branca & 64,7 & 1 & & 57,8 & 1 & \\
\hline Não-branca & 58,1 & $0,90(0,84-0,96)$ & & 52,4 & $0,91(0,84-0,98)$ & \\
\hline Tabagismo & & & 0,839 & & & 0,922 \\
\hline Não & 60,9 & 1 & & 54,6 & 1 & \\
\hline $\operatorname{Sim}$ & 60,3 & $0,99(0,90-1,08)$ & & 54,9 & $1,00(0,91-1,11)$ & \\
\hline Situação conjugal & & & 0,166 & & & 0,109 \\
\hline Solteiro (a) & 61,4 & 1 & & 55,3 & 1 & \\
\hline Outros & 56,7 & $0,92(0,82-1,03)$ & & 49,8 & $0,90(0,79-1,02)$ & \\
\hline Trabalhador & & & $<0,001$ & & & $<0,001$ \\
\hline Não & 65,5 & 1 & & 59,8 & 1 & \\
\hline Sim & 55,7 & $0,85(0,79-0,91)$ & & 48,9 & $0,82(0,75-0,89)$ & \\
\hline Reside com a família & & & 0,393 & & & 0,776 \\
\hline Não & 58,2 & 1 & & 53,7 & 1 & \\
\hline Sim & 61,2 & $1,05(0,94-1,18)$ & & 54,7 & $1,02(0,90-1,15)$ & \\
\hline Tipo de moradia & & & 0,862 & & & 0,457 \\
\hline Casa & 60,1 & 1 & & 54,4 & 1 & \\
\hline Apartamento/Outros & 60,4 & $0,99(0,88-1,11)$ & & 57,1 & $1,05(0,93-1,18)$ & \\
\hline $\begin{array}{l}\text { Aconselhamento para } \\
\text { atividade física }\end{array}$ & & & 0,509 & & & 0,452 \\
\hline Não & 61,6 & 1 & & 55,2 & 1 & \\
\hline Sim & 60,1 & $0,98(0,91-1,05)$ & & 53,6 & $0,97(0,89-1,05)$ & \\
\hline Percepção da saúde & & & $<0,001$ & & & $<0,001$ \\
\hline Boa/Excelente & 58,2 & 1 & & 52,0 & 1 & \\
\hline Regular/Ruim & 66,5 & $1,14(1,06-1,22)$ & & 59,7 & $1,15(1,06-1,24)$ & \\
\hline
\end{tabular}

624 • Rev. bras. Educ. Fís. Esporte, São Paulo, v.25, n.4, p.619-29, out./ dez. 2011 
TABELA 3 - Análise ajustada para as medidas deníveis insuficientes deatividadefísica em jovens do ensino médio noturno de Florianópolis, SC e Recife, PE, 2007.

\begin{tabular}{|c|c|c|c|c|}
\hline \multirow[t]{2}{*}{ Variáveis (Nível) } & \multirow{2}{*}{$\begin{array}{c}\begin{array}{c}\text { AF nos últimos } \\
\text { sete dias }\end{array} \\
\text { RP (IC95\%) }\end{array}$} & \multicolumn{3}{|c|}{$\begin{array}{l}\text { AF na semana } \\
\text { típica ou normal }\end{array}$} \\
\hline & & $\mathbf{p}^{*}$ & RP (IC95\%) & $\mathbf{p}^{*}$ \\
\hline Sexo (1) & & $<0,001$ & & 0,004 \\
\hline Masculino & 1 & & 1 & \\
\hline Feminino & $1,15(1,07-1,24)$ & & $1,12(1,04-1,22)$ & \\
\hline Idade (1) & & 0,481 & & 0,161 \\
\hline 15 a 19 anos & 1 & & 1 & \\
\hline 20 a 24 anos & $0,97(0,90-1,05)$ & & $0,94(0,85-1,03)$ & \\
\hline Cor da pele (1) & & 0,005 & & 0,033 \\
\hline Branca & 1 & & 1 & \\
\hline Não-branca & $0,90(0,84-0,97)$ & & $0,91(0,85-0,99)$ & \\
\hline Tabagismo (2) & & 0,862 & & 0,843 \\
\hline Não & 1 & & 1 & \\
\hline $\operatorname{Sim}$ & $0,99(0,90-1,09)$ & & $1,01(0,91-1,12)$ & \\
\hline Situação conjugal (3) & & 0,133 & & 0,143 \\
\hline Solteiro (a) & 1 & & 1 & \\
\hline Outros & $0,92(0,82-1,03)$ & & $0,91(0,79-1,03)$ & \\
\hline Trabalhador (3) & & $<0,001$ & & $<0,001$ \\
\hline Não & 1 & & 1 & \\
\hline $\operatorname{Sim}$ & $0,85(0,80-0,92)$ & & $0,82(0,76-0,89)$ & \\
\hline Reside com a família (3) & & 0,768 & & 0,602 \\
\hline Não & 1 & & 1 & \\
\hline $\operatorname{Sim}$ & $1,02(0,90-1,15)$ & & $0,96(0,84-1,10)$ & \\
\hline Tipo de moradia (4) & & 0,797 & & 0,426 \\
\hline Casa & 1 & & 1 & \\
\hline Apartamento/Outros & $0,98(0,88-1,10)$ & & $1,05(0,93-1,18)$ & \\
\hline Aconselhamento para atividade física (5) & & 0,361 & & 0,304 \\
\hline Não & 1 & & 1 & \\
\hline Sim & $0,97(0,90-1,04)$ & & $0,96(0,88-1,04)$ & \\
\hline Percepção da saúde (6) & & 0,002 & & 0,003 \\
\hline Boa/Excelente & 1 & & 1 & \\
\hline Regular/Ruim & $1,12(1,04-1,20)$ & & $1,13(1,04-1,22)$ & \\
\hline
\end{tabular}

$\mathrm{T}=$ Teste de Wald para heterogeneidade; $\mathrm{RP}=$ razão de prevalência. 


\section{Discussão}

Os resultados do presente estudo evidenciaram que a medida de atividade física nos últimos sete dias que antecederam a coleta de dados e durante uma semana típica ou normal estimam com diferenças significativas a prevalência de níveis insuficientes de atividade física. Entretanto, os fatores associados e a magnitude das medidas de associação foram semelhantes para as duas medidas.

Possivelmente, este é um dos primeiros estudos a comparar prevalências de níveis insuficientes de atividade física e alguns fatores associados determinados pela medida de atividade física considerando as atividades praticadas nos últimos sete dias e durante uma semana típica ou normal. Outro aspecto positivo é o envolvimento de jovens de duas cidades brasileiras com características socioculturais e ambientais distintas, Florianópolis (regiāo Sul) e Recife (região Nordeste).

Embora seja um aspecto importante, pouco se tem discutido sobre as implicaçôes do período de referência adotado, quando se avalia os níveis de atividade física, sobre as estimativas de prevalência de atividade física e possíveis fatores associados. $\mathrm{O}$ período de referência representa o intervalo de tempo que será utilizado para que os sujeitos recordem as atividades físicas praticadas (por exemplo, último dia, semana, mês, ano). A maioria dos questionários adota uma semana como período de referência (Armstrong \& Welsman, 2006; Kohl, Fulton \& Caspersen, 2000; Sirard \& Pate, 2001), porém não há consenso em relação à utilização dos últimos sete dias ou de semana típica ou normal.

A prevalência de níveis insuficientes de atividade física foi mais elevada ( $\cong 6$ pontos percentuais) quando a medida de atividade física considerou as atividades praticadas nos últimos sete dias, mesmo depois de se estratificar a análise por município. Contudo, não é possível afirmar que os resultados serão similares quando forem utilizados questionários que mensuram as atividades físicas praticadas em diferentes contextos.

É importante considerar possíveis efeitos da sazonalidade sobre esses dois períodos de referência. Como os dados foram coletados em um período curto de tempo (um mês) e durante o verão, neste estudo os possíveis efeitos da sazonalidade sobre essas duas medidas talvez não tenham sido evidenciados.

Apesar da magnitude das diferenças entre as prevalências de níveis insuficientes de atividade física, produzidas por essas duas medidas ter sido pequena (cerca de seis pontos percentuais), adotar os últimos sete dias ou uma semana típica, como período de referência, parece resultar em diferentes prevalências de níveis insuficientes de atividade física. Estes resultados sugerem que na comparação de prevalências de níveis insuficientes de atividade física entre diferentes estudos, é importante considerar, além do instrumento de medida (Gilson, Cooke \& Mahoney, 2001; Pate, Freedson, Sallis, Taylor, Sirard, Trost \& Dowda, 2002) e o ponto de corte adotado (PATE et al. 2002), o período de referência utilizado para recordar as atividades físicas.

$O$ fato de a atividade física ser um comportamento que apresenta grandes variaçōes (JAGO, ANDERSON, BARANOWSKI \& WATSON, 2005), na maioria dos sujeitos, a medida de atividade física nos últimos sete dias e durante uma semana típica ou normal podem fornecer diferentes informaçōes. A primeira representa uma medida das atividades físicas recentes ou atuais e a segunda uma medida das atividades físicas habitualmente praticadas (KRISKA \& CASPERSEN, 1997). Dessa forma, exceto quando a atividade física atual for igual à típica ou normal, diferenças entre as prevalências de níveis insuficientes de atividade física entre essas duas medidas deverão ser esperadas. Em decorrência da ausência de um critério de referência não foi possível avaliar a precisão das estimativas de níveis insuficientes de atividade física produzidas pelas medidas analisadas neste estudo.

Tanto na análise bruta quanto na análise ajustada, os fatores associados a níveis insuficientes de atividade física (sexo, cor da pele, trabalho, percepção de saúde), o sentido (associação positiva ou negativa) e a magnitude das medidas de associação foram similares para a medida de atividade física nos últimos setes dias e em uma semana típica ou normal (TABELA 3). Resultados semelhantes também foram encontrados para análise estratificada por município (dados não publicados). Ou seja, não há diferenças significativas (interseção do IC95\%) para prevalência de níveis insuficientes de atividade física e sua relação com fatores associados analisados entre a medida considerando as atividades físicas praticadas na última semana e durante uma semana típica ou normal.

Utilizar os últimos sete dias ou uma semana típica como referência para determinar os níveis de atividade física parece não influenciar na identificação de alguns fatores associados a níveis insuficientes de atividade física. Uma possível explicação para esses achados pode estar nas características dos fatores que foram analisados: fatores não modificáveis ou com elevado grau de dificuldade de serem modificados. Pode-se também 
considerar que para a maioria dos sujeitos analisados neste estudo, as atividades físicas recentes ou atuais correspondiam, em grande parte, às atividades físicas habituais. Também não se pode descartar a possibilidade dessas duas medidas apresentarem limitaçôes em discriminar com precisão as atividades físicas atuais das habituais.

É importante destacar que essas medidas apresentam vantagens e desvantagens que precisam ser consideradas. Acredita-se que os questionários que utilizam os últimos sete dias como período de referência permitam que os jovens recordem com maior precisão as atividades físicas praticadas, minimizando os problemas de recordação das informações solicitadas (Baranowski, 1988; McKenna, Foster \& Page, 2004). Entretanto, considerando que a atividade física é um comportamento que apresenta grandes variaçōes ao longo dos dias (JAGO et al., 2005), essa medida pode não refletir as atividades físicas que são habitualmente praticadas (KRISKA \& CASPERSEN, 1997).

Em contrapartida, quando se utiliza uma semana típica ou normal como período de referência, em tese, estão sendo mensuradas as atividades físicas que são habitualmente praticadas pelos sujeitos (Kriska \& Caspersen, 1997). Isso pode reduzir o impacto sobre as estimativas dos níveis de atividade física causado pelas variações que ocorrem nesse comportamento ao longo dos dias. Por outro lado, isso aumenta as chances de erros de estimativa, em decorrência da necessidade de se ter que combinar as variações ocorridas nas atividades físicas praticadas (JaGO et al., 2005; PATE et al., 2002). Além disso, essa medida mostra-se mais suscetível a viés de memória (BARANOWSKI, 1988), e há uma falta de informação e de padronização sobre o tempo de prática vs. interrupção que caracteriza um padrão habitual.

Este estudo apresenta algumas limitaçôes que devem ser levadas em consideração. Uma delas foi o fato de ter incluído apenas estudantes do ensino médio de escolas públicas e que estudavam no turno noturno. Normalmente, estes sujeitos apresentam características particulares em relação ao desfecho e para alguns fatores associados que foram analisados. Não se sabe se esses resultados seriam semelhantes caso o estudo fosse desenvolvido em uma amostra representativa da população adolescente.

Outra limitação que merece ser destacada é a ausência de uma medida que possa ser considerada como padrão-ouro para mensurar o nível de atividade física. Dessa forma, este estudo não pode testar qual das medidas analisadas (os últimos sete dias ou uma semana típica), produz estimativas mais precisas de prevalência de níveis insuficientes de atividade física, quando se utiliza um questionário simplificado. Entretanto, essa não é uma tarefa fácil, pois as medidas objetivas de atividade física mensuram atividades físicas atuais (mais recentes) o que exigiria a sua aplicação por várias semanas nos mesmos sujeitos, tornando-se praticamente inviável em termos de logística.

A utilização de diferentes períodos de referência para recordar as atividades físicas praticadas produz prevalências de níveis insuficientes de atividade física diferentes, sendo mais elevadas quando as atividades praticadas nos últimos sete dias são consideradas em comparação àquelas praticadas durante uma semana típica ou normal. As variáveis que se mostraram associadas a níveis insuficientes de atividade física; bem como, o sentido e a magnitude das medidas de associação foram similares para as medidas de atividade física nos últimos sete dias e em uma semana típica ou normal. Esse comportamento se manteve mesmo quando os dados foram analisados separadamente por cidade, indicando que esse padrão de comportamento parecer persistir mesmo entre adolescentes expostos a diferentes características socioculturais e ambientais (Recife, PE e Florianópolis, SC).

Recorrer aos últimos sete dias ou a uma semana típica como período de referência para mensurar as atividades físicas praticadas pelos adolescentes, a partir da utilização de questionários simplificados, tende a gerar prevalências de níveis insuficientes de atividade física diferentes, porém fatores associados semelhantes.

\section{Abstract}

Prevalence and associated factors to insufficient level physical activity and in youth from two city of Brazil: last seven days or typical week

This study compared the prevalence rates of insufficient physical activity levels and of certain related factors determined for using physical activity measure during in the past seven days, and in a typical week. This is a cross-sectional analysis (baseline data) of a school-based randomized trial (the Saude na Boa project), 
including 2,096 students (15-24 years of age; 55.6\% females) from 10 schools in Florianopolis and 10 schools in Recife, pair-matched by size and location. Data were collected by a previously validated questionnaire. Individuals who reported performing moderate to vigorous intensity physical activities lasting $\geq 60$ minutes, $\geq 5$ days per week were classified as "active" and the remainders of the sample were classified as "insufficiently active". This classification was performed on the basis of the activity performed during two reference periods: last seven days and typical week. The measure of the physical activities considering the period reference of last the seven days resulted in higher prevalence of insufficient levels of physical activity in comparison to the measure considering the reference of typical week $(60.8 \%$ vs. $54.6 \% ; p<0.001)$. The factors associated with and insufficient level of physical activity, and the magnitudes of the measures of association were similar for two reference periods. Independent of the reference period adopted for recall the physical activities, the insufficient levels of physical activity had been identified to the same associated factors, with a little variation in the magnitude of the measures of association. To use past seven days or typical week as reference period to measure the levels of physical activity seems to generate different prevalence of insufficient levels of physical activity, but same associated factors.

UnITERMs: Young; Physical activity measure; Prevalence; Associated factors.

\section{Referências}

ARMSTRONG, N.; WELSMAN, J. R. The physical activity patterns of European youth with reference to methods of assessment. Sports Medicine, Auckland, v.36, p.1067-86, 2006.

BARANOWSKI, T. Validity and reliability of self-report measures of physical activity: an information-processing perspective. Research Quarterly for Exercise and Sport, Washington, v.59, p.314-27, 1988.

BARROS, A.J.; HIRAKATA, V.N. Alternatives for logistic regression in cross-sectional studies: an empirical comparison of models that directly estimate the prevalence ratio. BMC: Medical Research Methodology, London, v.3, n.21, 2003. Disponível em: <http://www.biomedcentral.com/1471-2288/3/21>.

BARROS, M.V.G.; NAHAS, M.V.; HALLAL, P. R.C.; FARIAS JÚNIOR, J.C.; FLORINDO, A.A.; HONDA, S.S. Effectiveness of a school-based intervention on physical activity for high-school students in Brazil: the Saúde na Boa projects in Brazil: The Saúde na Boa Project. Journal of Physical Activity \& Health, Champaign, v.6, p.163-9, 2009.

BIDDLE, S.; SALLIS, J.F.; CAVILL, N.A. Young and active? Young people and health enhancing physical activity: evidence and implication. London: Health Education Authority, 1998.

BUTCHER, K.; SALLIS, J.F.; MAYER, J.A.; WOODRUFF, S. Correlates of physical activity guideline compliance for adolescents in 100 U.S. cities. Jornal of Adolescent Health, New York, v.42, p.360-8, 2008.

CENTERS FOR DISEASE CONTROL AND PREVENTION (CDC). Youth risk behavior surveillance: United States, 2007. Surveillance Summaries. Morbidity and Mortality Weekly Report, Atlanta, v.57, n.SS-4, 2008.

CHINAPAW, M.J.M.; MOKKINK, L.B.; VAN POPPEL, M.N.M.; VAN MECHELEN, W.; TERWEE, C.B. Physical activity questionnaire for youth: a systematic review of measurement properties. Sports Medicine, Auckland, v.40, n.7, p.539-63, 2010. GILSON, N.D.; COOKE, C.B.; MAHONEY, C.A. A comparison of adolescent moderate-to-vigorous physical activity participation in relation to a sustained or accumulation criterion. Health Education Research, Washington, v.16 p.335-41, 2001. HALLAL, P.C.; DUMITH, S.D.; BASTOS, J.P.; REICHERT, F.F.; SIQUEIRA, F.V.; AZEVEDO, M.R. Evolução da pesquisa epidemiológica em atividade física no Brasil: revisão sistemática. Revista de Saúde Pública, São Paulo, v.41, p.453-60, 2007. JAGO, R.; ANDERSON, C.B.; BARANOWSKI, T.; WATSON, K. Adolescent patterns of physical activity: differences by gender, day, and time of day. American Journal of Preventive Medicine, Amsterdam, v.28, p.447-52, 2005.

KOHL, H.W.; FULTON, J.E.; CASPERSEN, C.J. Assessment of physical activity among children and adolescents: a review and synthesis. Preventive Medicine, New York, v.31, p.S54-S76, 2000.

KRISKA, M.K.; CASPERSEN, C.J. Introduction to a collection of physical activity questionnaire. Medicine and Science in Sports and Exercise, Madison, v.29, p.S5-S9, 1997.

MALDONA, G.; GREELAND, S. Simulation Study of confounder-selection strategies. American Journal of Epidemiology, Baltimore, v.138, p.923-36, 1993.

McKENNA, J.; FOSTER, L.J.; PAGE, A. Exploring recall of physical activity in young people using qualitative interviewing. Pediatric Exercise Science, Champaign, v.16, p.5-14, 2004. 
NAHAS, M.V.; BARROS, M.V.G.; ASSIS, M.A.A.; HALLAL, P.R.C.; FLORINDO, A.A.; KONRAD, L.M. Methods and participant characteristics of a randomized intervention to promote physical activity and healthy eating among brazilian high school students: The Saúde na Boa Project. Journal Physical Activity and Health, Champaign, v.6, n. 153-62, 2009. NAHAS, M.V.; BARROS, M.V.G.; FLORINDO, A.A.; FARIAS JUNIOR, J.C.; HALLAL, P.C.; KONRAD, L.; BARROS, S.S. H.; ASSIS, M.A.A. Reprodutibilidade e validade do questionário Saúde na Boa para avaliar atividade física e hábitos alimentares em escolares do ensino médio. Revista Brasileira de Atividade Física e Saúde, Londrina, v.13, p.10-8, 2007. NAHAS, M.V.; BARROS, M.V.G.; GOLDFINE, B.D.; LOPES, A.S.; HALLAL, P.C.; FARIAS JÚNIOR, J.C.; OLIVEIRA, E.S. Physical activity and eating habits in public high schools from different regions in Brazil: the Saúde na Boa project. Revista Brasileira de Epidemiologia, São Paulo, v.12, p.270-7, 2009.

PATE, R.R.; FREEDSON, P.; SALLIS, F.J.; TAYLOR, W.C.; SIRARD, J.; TROST, S.G.; DOWDA, M. Compliance with physical activity guidelines: prevalence in a population of children and youth. Annals of Epidemiology, New York, v.12, p.303-8, 2002. PROCHASKA, J.J.; SALLIS, J.F.; LONG, B. A physical activity screening measure for use with adolescents in primary care. Archives of Pediatrics \& Adolescent Medicine, Chicago, v.155, p.554-9, 2001.

PROCHASKA, J.J.; ZABINSKI, M.F.; CALFAS, K.J.; SALLIS, J.F.; PATRICK, K. PACE+ Interactive communication technology for behavior change in clinical settings. American Jornal of Preventive Medicine, Amsterdam, v. 19, p.127-31, 2000. SALLIS, J.F.; SAELENS, B.E. Assessment of physical activity by self-report: status, limitations, and future directions. Research Quarterly for Exercise and Sport, Washington, v.71, p.1-14, 2000.

SIRARD, J.R.; PATE, R.R. Physical activity assessment in children and adolescents. Sports Medicine, Auckland, v.31, p.439-54, 2001.

STRONG, W.B.; MALINA, R.M.; BLIMKIE, C.J.R.; DANIELS, S.R.; DISHMAN, R.K.; GUTIN, B.; HERGENROEDER, A.C.; MUST, A.; NIXON, A.A.; PIVARNIK, J.M.; ROWLAND, T.; TROST, S.; TRUDEAU, F. Evidence based physical activity for school-age youth. Journal of Pediatric, Cincinnati, v.146, p.732-7, 2005.

VARO, J.J.; MARTINEZ-GONZÁLEZ, M.A.; IRALA-ESTÉVEZ, J.; KEARNEY, J.; GIBNEY, M.; MARTINEZ, J.A. Distribution and determinants of sedentary lifestyles in the European Union. International Journal of Epidemiology, London, v.32, p.138-46, 2003.

VICTORA, C.G.; HUTTLY, S.R.; FUCHES, S.C.; OLINTO, M.T. The role of conceptual frameworks in epidemiological analysis: a hierarquical approachI. International Epidemiological Association, London, v.26, p.224-7, 1997. WORLD HEALTH ORGANIZATION (WHO). Inequalities in young people's health. Copenhagen: WHO Regional Office for Europe, 2008. Disponível em: <http://www. euro.who.int>. Acesso em: 20 fev. 2008.

\section{Agradecimentos}

O estudo foi financiado pelo Projeto Healthy Lifestyle, Healthy People, do consórcio composto pelo International Life Sciences Institute (ILSI), pela Organização Panamericana de Saúde (OPAS) e pelo CDC (Centers for Disease Control and Prevention).

O estudo também recebeu apoio do Conselho Nacional de Desenvolvimento Científico e Tecnológico (CNPq), na forma de bolsa de produtividade em pesquisa, e das Universidades Federal de Santa Catarina e de Pernambuco.

Os autores agradecem de forma especial aos estudantes e professores, diretores de escolas e Secretarias de Educação do Estado de Santa Catarina e Pernambuco.

ENDEREÇO

J osé Cazuza de Farias J únior Departamento de Educação Física Universidade Federal da Paraíba Cidade Universitária 58059-900 - J oão Pessoa - PB - BRASIL e-mail: jcazuzajr@hotmail.com
Recebido para publicação: 03/01/2011

Revisado: 19/07/ 2011

Aceito: 25/08/2011 